\title{
MASS SPECTROMETER FOR ACCURATE HYDROGEN ISOTOPIC ANALYSES
}

P. CHASTAGNER

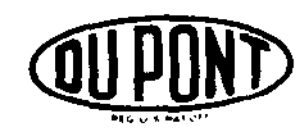

SAVANNAH RIVER LABORATORY AIKEN, SOUTH CAROLINA 29801 


\section{NOTICE}

This report was prepared as an account of work sponsored by the United States Government. Neither the United States nor the United States Department of Energy, nor any of their contractors, subcontractors, or their employees, makes any warranty, express or implied or assumes any legal liability or responsibility for the accuracy, completeness or usefulness of any information, apparatus, product or process disclosed, or represents that its use would not infringe privately owned rights.

Printed in the United States of Americe

Available from

Netional Technicel Information Service

U.S. Department of Commerce

5285 Port Roval Rosd

Springfield, Virginia 22161

Price: Printed Copy $\$ 4.50 ;$ Microfiche $\$ 3.00$ 
DP-1492

Distribution Category, UC-4

\section{MASS SPECTROMETER FOR ACCURATE HYDROGEN ISOTOPIC ANALYSES}

by

P. Chastagner

Savannah River Laboratory

E. I. du Pont de Nemours \& Co.

Aiken, South Carolina 29801

Approved by

R. L. Folger, Research Manager

Analytical Chemistry Division

Publication Date: August 1978

E. I. DU PONT DE NEMOURS AND COMPANY SAVANNAH RIVER LABORATORY AIKEN, SOUTH CAROLINA 29801 PREPARED FOR THE U.S. DEPARTMENT OF ENEAGY UNDER CONTRACT AT $\{07 \cdot 2\}-1$ 


\section{ABSTRACT}

A theoretical study of the mass spectrometric analysis of mixtures of the hydrogen and helium isotopes shows that mass discrimination and low ion intensity in ion sources are the principal factors that limit accuracy. A virtual-image ion source with short focal length and high brightness avoids these limitations. Suitable analyzers and accessory equipment are available. Development and construction of an ion source with the required characteristics are within the state of the art of mass spectrometry. A high-resolution mass spectrometer with a satisfactory ion source should routinely analyze mixtures of the hydrogen and helium isotopes accurately within $+0.5 \%$ relative $(99.7 \% \mathrm{CL})$ with ordinary operating techniques. Isotopic equilibrium, auxiliary helium analyses, and accurate standard would not be needed. Analyses accurate within $+0.3 \%$ relative ( $99.7 \% \mathrm{CL}$ ) could be made by using accurately, mixed standards. 


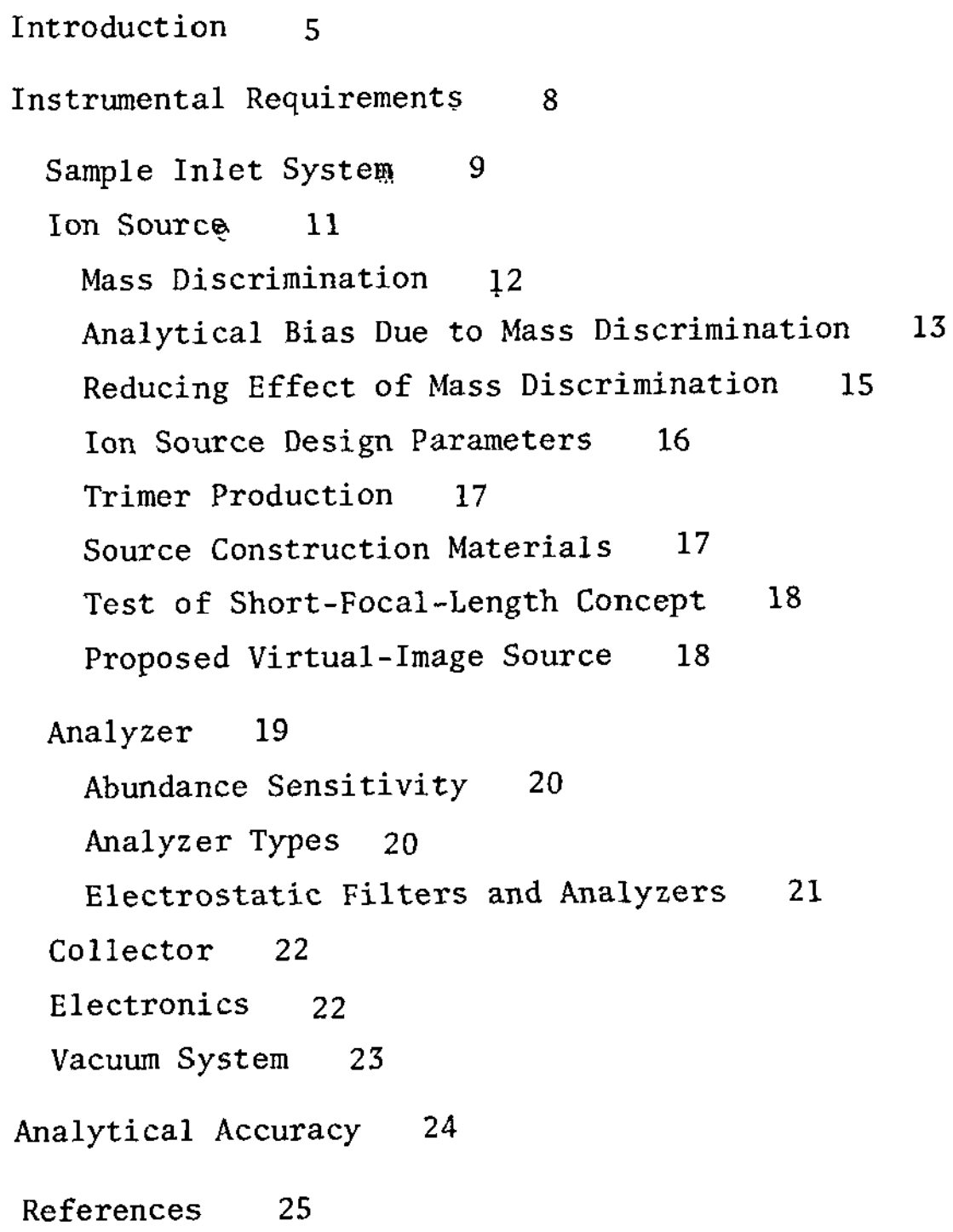




\section{LIST OF TABLES}

1 M/E Peaks of Analytical Importance in the Mass Range $2-6 \quad 6$

2 Mass Separation as a Function of Ion-Source Focal Length 13

\section{LIST OF FIGURES}

1 Determination of ${ }^{3} \mathrm{He}$ by Titanium-Sublimation Pumping 7

2 Sample Inlet System 10

3 Nier-Type Ion Source 11

4 Ideal Ion-Beam Profile 14

5 Deconvoluted Ion-Beam Profile Showing Mass Discrimination 14

6 Deconvoluted Ion-Beam Profile Showing Effect of Defocusing 16

7 Virtua1-Image-Type Ion Source 18

8 Amplifier Performance 23 


\section{INTRODUCTION}

Accurate mass spectrometric analyses of mixtures of the three hydrogen isotopes (D, $H$, and $T$ ) are difficult to make because the mixtures contain six molecular species and ${ }^{3} \mathrm{He}$ (from the decay of $\mathrm{T}$ ). The heaviest species has three times the mass of the lightest, and mass discrimination is particularly severe.

In a mixture that contains two species with the same mass number, smal1 mass differences and large concentration differences complicate the analysis. The mass difference between $\mathrm{HD}$ and ${ }^{3} \mathrm{He}$ is one part in 520 , while that between HT and $D_{2}$ at mass 4 is only one part in 980 . The concentration ratio of the two species is often more than $1000: 1$. The scatter of the more-abundant species interferes with the measurement of the less-abundant species. To separate the $\mathrm{D}_{2}-\mathrm{HT}$ doublet reliably, the resolution of the mass spectrometer should be at least one part in 1400 (50\% more than that required for peaks of equal size), and the abundance sensitivity should be at least 10,000.

Monatomic and polyatomic ions formed in the ion source (Table 1) further complicate the mass spectral analyses. The $\mathrm{T}^{+}$ and ${ }^{3} \mathrm{He}$ differ by only 1 part in 151,000 and probably cannot be separated with any practical analytical mass spectrometers. Fortunately, $\mathrm{T}^{+}$ion production is a small constant fraction of the $\mathrm{T}_{2}$, DT, and HT concentrations, and the error in correcting the ${ }^{3} \mathrm{He}$ mass peak is trivial. Trimer formation is a function of the pressure in the ion source and is usually very small.

Hydrogen isotopic mixtures can be analyzed with low $(<200)-$, medium (about 600)-, or high (>1400)-resolution mass eters. When either low- or medium-resolution mass spectrometers are used, the sample must be at equilibrium. The relative amounts of $\mathrm{D}_{2}$ and $\mathrm{HT}$ at mass 4 are calculated using the equilibrium constants published by Jones. ${ }^{1}$ When high resolution instruments are used, all of the species of interest are measured directly.

${ }^{3} \mathrm{He}$ content is measured directly with medium- and high-resolution instruments but must be measured by some other method, such as absorption of the hydrogen fraction of the sample on uranium or titanium, with low resolution instruments. This technique is relatively imprecise as shown in Figure 1 . The error in the ${ }^{3} \mathrm{He}$ determination causes a corresponding error in the HD value. The HD error is propagated directly to the HT value via the equilibrium calculation. 
TABLE 1

M/e Peaks of Analytical Importance in the Mass Range 2-6 Species

$M / e$

Resolution Required

$D^{+}$

$\mathrm{H}_{2}^{+}$

2.0140

${ }^{3} \mathrm{He}^{+}$

2.01565

3.01603

$\mathrm{T}^{+}$

$\mathrm{HD}^{+}$

3.01605

3.021825

$\mathrm{H}_{3}{ }^{+}$

3.023475

${ }^{4} \mathrm{He}^{+}$

4.00260

$\mathrm{HT}^{+}$

4.023875

$\mathrm{D}_{2}{ }^{+}$

4.028

$\mathrm{DT}^{+}$

5.03005

$\mathrm{T}_{2}{ }^{+}$

6.032

$\mathrm{D}_{3}{ }^{+}$

6.0420

1,220

$$
2.5
$$

155,000

520

1,830

3.5

160

980

4.5

5.5

610

a. Isotope masses-from-Handbook-of-Chemistry and Physics (56th ed.). Robert C. Weast, Ed. CRC Press, Cleveland, Ohio (1975). p. B-253.

$\begin{array}{llll}\text { Isotope } & \text { Mass, comu } & \text { Isotope } & \text { Mass, comu } \\ \mathrm{H} & 1.007825 & { }^{3} \mathrm{He} & 3.01603 \\ \mathrm{D} & 2.0140 & { }^{4} \mathrm{He} & 4.00260 \\ \mathrm{~T} & 3.01605 & & \end{array}$




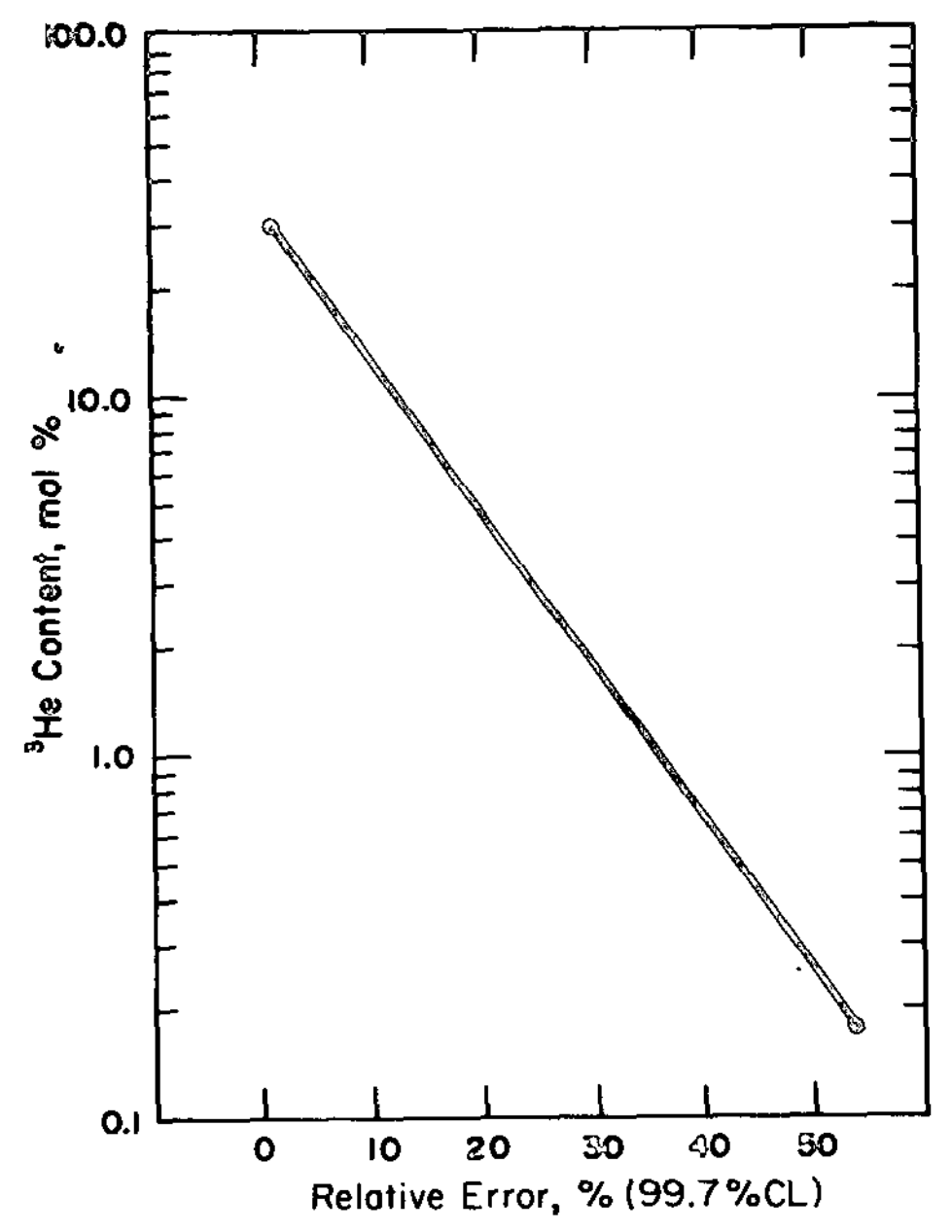

FIGURE 1. Determination of ${ }^{3} \mathrm{He}$ by Titanium-Sublimination Pumping

In some mixtures, as much as $1 \%$ of the total $\mathrm{T}$ can be present as HT when the sample is at equilibrium, but in fresh mixtures, the HT concentration can be several times its equilibrium value. If equilibrium is assumed, an erroneous value may be calculated for total $\mathrm{T}$.

No commercial mass spectrometer is available that can be used directly for accurate analyses of mixtures of the hydrogen isotopes. The best of the current commercial instruments are designed for organic analyses and cannot be used for quantitative hydrogen isotopic analyses without extensive modifications.

In the past, hydrogen isotopic analyses usually were made with commercially available low- or medium-resolution mass spectrometers. Equilibrium among the isotopic species was assumed 
for mixtures containing tritium, but was not usually verified. ${ }^{3} \mathrm{He}$ was either estimated from the mass spectrum or determined separately by an auxiliary technique. The limit of accuracy was about \pm 2 to $\pm 4 \%$ and was adequate for many programs.

The available high-resolution mass spectrometers were expensive and produced low ion intensity; they were difficult and time-consuming to operate and, judging by the work of Schott and Beau, ${ }^{2}$ were no more accurate than the less-expensive, lowresolution instruments.

Because no mass spectrometer suitable for the accurate analysis of mixtures of hydrogen and helium isotopes is available, a theoretical study of the mass spectrometry of the hydrogen isotopes was made to determine 1) instrumental requirements for accurate high-resolution analyses, and 2) whether or not construction of a suitable instrument is within the state of the art of mass spectrometry. The results of the theoretical study and the requirements for the desired instrument are given in this report.

\section{INSTRUMENTAL REQUIREMENTS}

A satisfactory high-resolution mass spectrometer for routine analy.sis of samples containing mixtures of hydrogen and helium isotopes should have the following characteristics:

- Sample size <2 cc atm.

- Ion-beam intensity about $1 \times 10^{-9}$ A for the major ion species at the collector. The beam should be representative of the sample and be free of mass discrimination.

- A resolving power of 2,000 at mass 4 .

- An abundance sensitivity of at least 10,000 for the HT- $\bar{D}_{2}$ doublet.

- Ion-current measurement accurate within $0.1 \%$ for the major species and $1 \%$ for the minor species.

- Retention of calibration within $0.2 \%$ for a period of several hours.

- Easy operation by nontechnical personnel.

- High reliability with minimum maintenance.

- An analytical rate of at least six samples per hour. 
The mass spectrometer is a combination of a number of components, each of which must be designed both to do its own job well and to be compatible with the other components of the instrument system. The design and role of each of the various parts of the mass spectrometer system is discussed in the sections that follow. This discussion is limited to sector-type mass spectrometers because they have been studies more extensively than have any other type. Sector-type instruments are modular and easy to design, and suitable analyzers and auxiliary equipment are commercially available.

\section{Sample Inlet System}

The sample inlet system is a critical part of the system because it controls the amount of gas that flows into the ion source. A refined inlet system of the type shown in Figure 2 has proven to be satisfactory in use at the Savannah River Plant. The system has the following features.

- A large sample volume to minimize the pressure drop caused by flow through the molecular leak ( $5 \mathrm{~L}$ with the molecular leak specified below).

- All-stainless steel construction with metal gasketed seals.

( ) Bakeable to at least $250^{\circ} \mathrm{C}$ to minimize sample contamination, memory, and leakage.

- Simple design to minimize the number of valves and leakage problems.

- All metal valves installed with seats facing the sample volume to ensure that sample volume will remain constant from sample to sample.

Temperature control is critical because the gas content of the inlet system at any given pressure is inversely proportional to the temperature. When the temperature of the entire inlet system including the molecular leak is held constant to within $\pm 0.1^{\circ} \mathrm{C}$, the analytical error due to temperature variations is insignificant $(<0.03 \%)$

The precision and accuracy of absolute determinations cannot be better than the precision, linearity, and reproducibility of the pressure-measuring device. The device must have an overall limit of error of $<+0.2 \%$. Capacitance manometers that meet this error specification are available. Sonar manometers, with differential pressure cells to isolate the mercury, are equally 


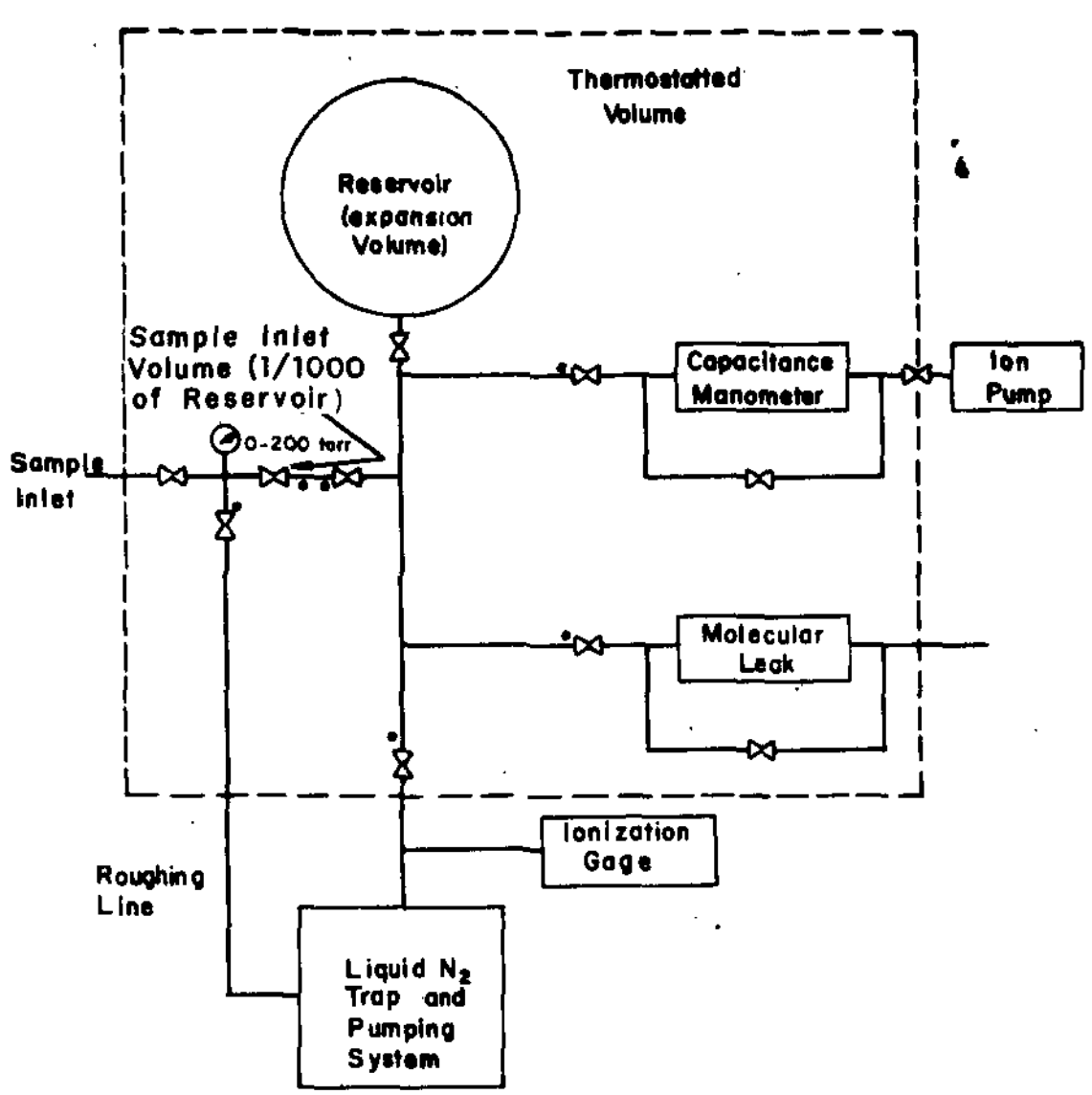

FIGURE 2. Sample Inlet System

precise and are more accurate than capacitance manometers. Sonar manometers could be used but they are more expensive, quite cumbersome, and difficult to install. Because repeatability and linearity are critical and absolute accuracy is not, the sonar manometer does not have any significant advantage over the capacitance-manometer-for-this-app1ication.

The same molecular leak must be used for all calibrations and sample analyses. The smallest leak that will supply an adequate amount of gas to the ion source should be used to minimize sample depletion during the analysis. A molecular leak with a flow rate of $0.2 \mathrm{cc} / \mathrm{sec}$ of less is satisfactory.

The manifold and pumping system of the inlet system should be sized to pump the system to a pressure of $<1 \times 10^{-7}$ torr during bakeout and between sample analyses to minimize memory and crosscontamination. 


\section{Ion Source}

No available ion source has the ion intensity and freedom from mass discrimination required for accurate high-resolution analyses of mixtures of the hydrogen and helium isotopes. A major development effort will be required to design and build a satisfactory source. The ion source must emit a stable, well-defined ion beam with an intensity of about $1 \times 10^{-9} \mathrm{~A}$ (measured at the collector) for the major isotopic species, so that the minor isotopic species can be measured accurately. This high ion current improves the precision and accuracy of ion current measurement, permits the use of the fast amplifiers needed to meet the required analysis rate, and simplifies the operation of the instrument. The ion beam width must be small, well defined, and free of mass discrimination and memory.

The sample should not equilibrate in the ion source and trimer production should be very low. The source must be very efficient to minimize sample consumption and to maintain the pressure in the ion chamber at a reasonable level. The sensitivity should be uniform to within $0.5 \%$ for all of the isotopic species.

In a typical electron-impact type ion source (Figure 3), electrons emitted by a heated filament are accelerated through an ionization chamber where they collide with sample molecules to form ions. The ions are extracted from the chamber, focused into a beam, and are accelerated toward the analyzer by a fixed high potential between the ionization chamber and the source exit slit.* The source lens system focuses the ion beam on the source exit slit. This slit defines the width of the ion beam and is the object image of the mass analyzer.

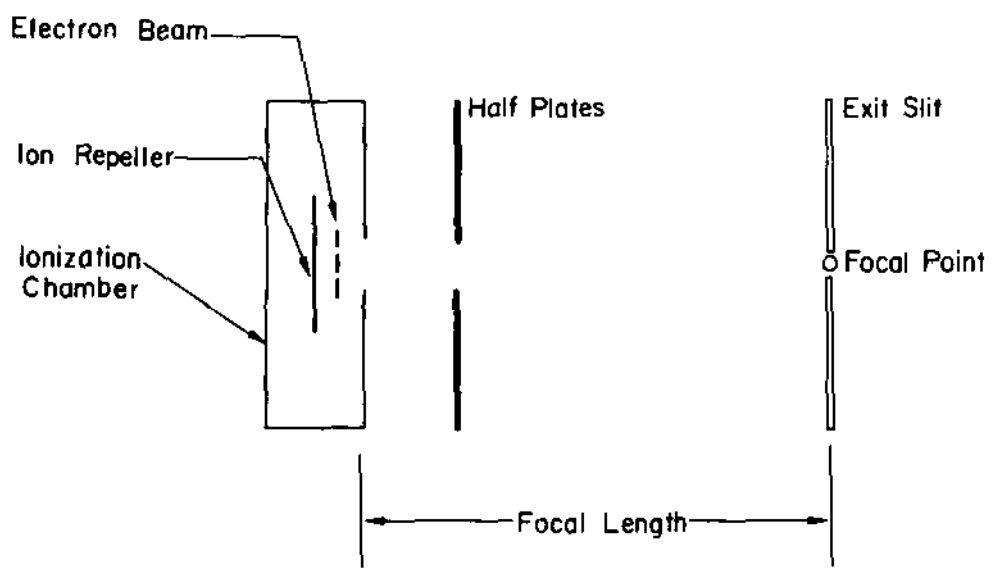

FIGURE 3. Nier-Type Ion Source

* A fixed accelerating voltage and magnetic scanning are used to eliminate the sensitivity changes and mass discrimination associated with voltage scanning. 
The electron beam in the ionization chamber is defined by a slit in front of the filament and is collimated along the length of the ionization chamber exit slit by a magnetic field parallel to the electron path (perpendicular to the plane of Figure 3). A portion of the electron beam passes through a small hole and impinges on an electrode called a trap. The electron current at the trap is used to control the intensity of the electron beam. The collimating magnetic field is required to make the electron density in the ion-extraction zone high enough for efficient ion production. Unfortunately, this field makes the ion source a miniature mass spectrometer and causes mass discrimination.

\section{Mass Discrimination}

Mass discrimination is especially severe for the hydrogen isotopes because of their low masses, ease of deflection, and high relative mass ratios. The mass separation in the source depends on the accelerating potential, source magnet field strength, and the focal length of the source. The interrelationship of the mass, $M$, charge, e, magnetic field strength, $H$, accelerating potential, $V$, and the radius of curvature of the ion path, $R$, is shown in the equation

$$
\frac{M}{e}=K \frac{H^{2} R^{2}}{V}
$$

where $K$ is a constant. The field of the collimating magnet is fixed. The highest practical accelerating potential (8-10 kV) should be used to maximize the radius of curvature of the ion paths and reduce the mass separation at the source exit slit. High accelerating potentials also extract and focus ions more efficiently from ion sources and improve their performance.

Mass discrimination can be reduced by making the focal length of the ion source very short. The longer the source, the greater the mass separation at the exit slit. The amount of discrimination depends on the mass separation and the size of the exit slit. The mass separations caused by a typical 400-Gauss collimating magnet in ion sources with three different focal lengths operating at an accelerating voltage of $8 \mathrm{kV}$ is shown in Table 2 .

For accurate ion intensity measurements, the collector slit must be at least as wide as the incident ion beam. With the present state-of-the-art of mass spectrometry, it is unlikely that a $1 \times 10^{-9}$-A ion beam free of major aberrations can be formed with a width less than 50-75 $\mu \mathrm{m}$. Therefore, a source-exit slit about $50 \mathrm{\mu m}$ wide is required. Because the mass 2-6 separation values in Table 2 are large compared with the $50-\mu \mathrm{m}$ slit width, only the 
mass focused on the center of the slit is transmitted efficiently. The other masses are off-center and are clipped or "discriminated against." The mass separation in the longer sources is greater than the 50-um exit-slit width and mass discrimination is severe. If $\mathrm{H}_{2}$, which is only a minor component in $\mathrm{D}-\mathrm{T}$ mixtures, is totally ignored, each source could be focused to optimize the transmission of ions in the mass 4-6 range. The mass discrimination in the 10-mm focal length source would still be severe. Reducing the collimating field strengh (if it could be done without sacrificing sensitivity) by a factor of 10 would make the mass discrimination minor in the $45-\mathrm{mm}$ source and would essentially eliminate discrimination in the 10-mm source. Total elimination of the collimating magnetic field would be impractical because the sensitivity loss would be severe.

\section{TABLE 2}

Mass Separation as a Function of Ion-Source Focal Length ${ }^{a}$

\begin{tabular}{lrr} 
Focal Length, & \multicolumn{2}{c}{ Mass Separation, $\mu m$} \\
& $2-6$ & $4-6$ \\
10 & 40 & 14 \\
45 & 350 & 100 \\
75 & 600 & 200
\end{tabular}

a. Conditions: accelerating voltage, $8 \mathrm{kV}$; collimating magnetic field, 400 Gauss (decreasing to zero $75 \mathrm{~mm}$ from magnet center).

\section{Analytical Bias Due to Mass Discrimination}

To obtain a reasonably accurate sensitivity value for DT (for which there is no pure gas standard), users of low and mediumresolution mass spectrometers customarily adjust the instruments to make the $D_{2}$ and $T_{2}$ sensitivities nearly equal. The DT sensitivity is then taken to be the average of the $D_{2}$ and $T_{2}$ sensitivities. If there were no mass discrimination, and intensity profile of the ion beam at the exit slit would be a single normally distributed curve as shown in Figure 4. Unfortunately, there is some mass separation in the ion source. When the partially separated $D_{2}$ and $T_{2}$ ions are focused for equal sensitivity, they are clipped slightly by the slit edges as shown in the deconvoluted ion profile in Figure 5. This focusing procedure centers the DT ions on the exit slit and the DT is not clipped. The transmission is higher for the DT than for the $D_{2}$ and $T_{2}$ ions and the true DT sensitivity is higher than the average value used. The bias is small in low-resolution 
instruments with their large exit slits but is severe in highresolution instruments where sma11 exit slits are used. This bias is a hidden error that cannot be measured or corrected without accurate mixed standards. Accurate independent analyses based on pure gas standardization alone would be impossible.

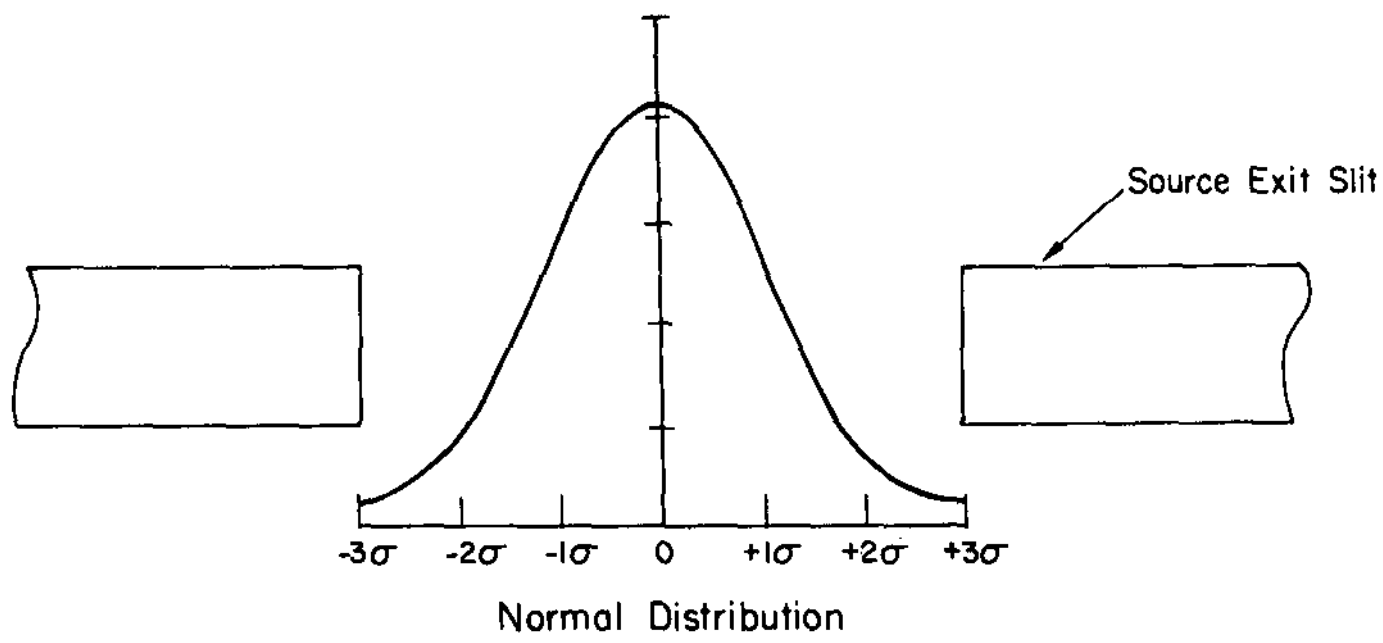

FIGURE 4. Idea] Ion-Beam Profile

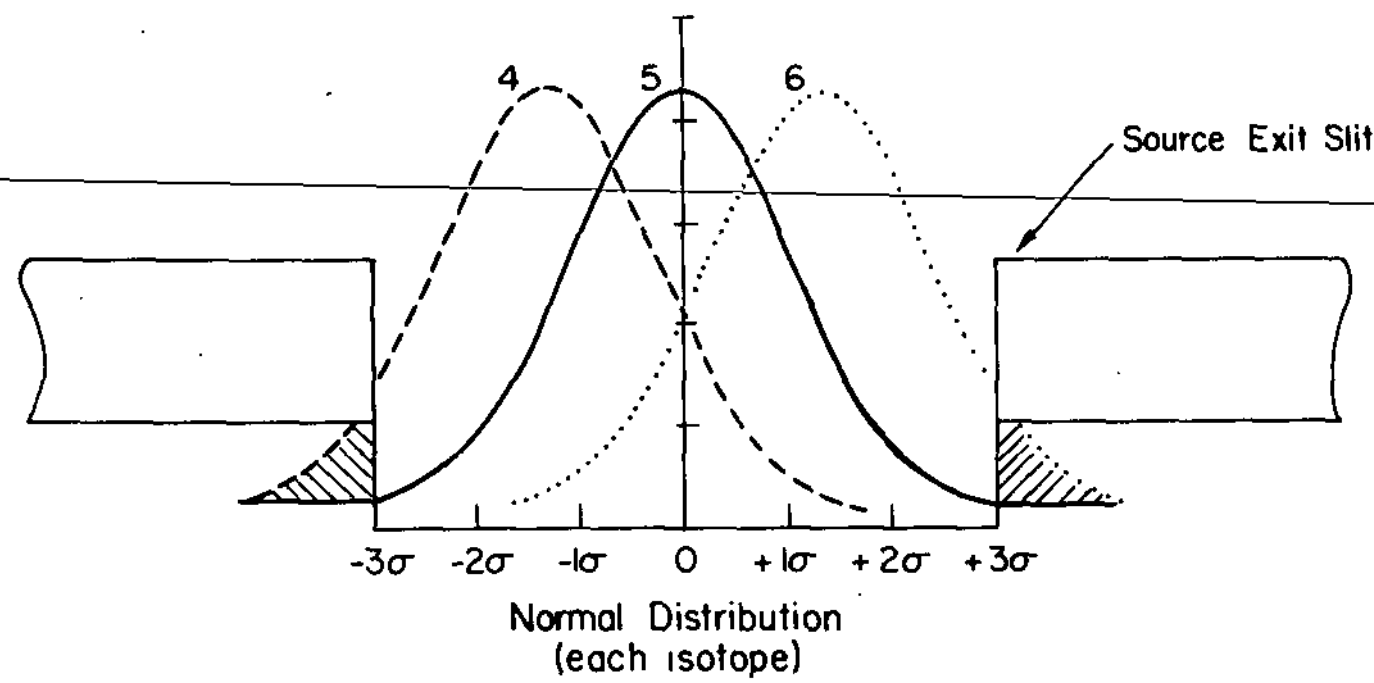

FIGURE 5. Deconvoluted Ion-Beam Profile Showing Mass Discrimination 


\section{Reducing Effect of Mass Discrimination}

Two ways in which the effects of mass discrimination in an ion source can be reduced are 1) refocusing the source for each mass as it is measured, the 2) defocusing the source. The focusing plate in the ion lens is normally split into two half plates, as shown in Figure 3, to provide lateral shifting of the ion beam so it can be focused on the center of the exit slit. Where there is severe mass discrimination, as can be the case with mixtures of the hydrogen isotopes, the focal point of each mass is displaced from the others across the exit slit as shown in Figure 5 . Only one species can be centered on the exit slit at any one time. The voltage differential between the half plates required to center each of the masses on the exit slit can be determined by carefully refocusing for each mass during the calibration procedure. Because a11 of the sensitivities are determined with the individual ion species centered on the exit slit, the DT sensitivity can be determined from the curve generated by plotting all of the measured sensitivities as a function of mass. If the curve is a straight line, the DT sensitivity will be the average of the $D_{2}$ and $T_{2}$ sensitivities. When samples are analyzed, it is necessary to readjust the ion source for optimum focusing of each mass as it is scanned. This is a tedious procedure when applied manually with a slow scan. It is difficult, if not impossible, to accomplish with a fast scan. An automatic control such as a computer or a microprocessor would be required to apply this procedure to a large number of routine samples. The calibration difficulties, manpower requirements, and the need for automatic control makes this alternative both technically difficult and uneconomical.

Defocusing is a more attractive way to reduce mass discrimination in the ion source. The intensity profile of each of the ion beams is spread so the top of each profile is almost flat and the transmission is almost the same for each ion species. For example, the set of ion beam profiles shown in Figure 5 becomes the set shown in Figure 6 and mass discrimination is almost completely eliminated. Unfortunately, sensitivity is lost when the source is defocused. In the examples illustrated in Figures 4 and 5 , the beam is focused so the $+3 \sigma$ ion distribution is equal to the exit slit width and $99.7 \%$ of the ions pass through the slit. When the source is defocused enough to eliminate mass discrimination, the $+3 \sigma$ ion distribution is 6 times the width of the exit slut and on $\overline{1} y+0.5 \sigma$ or $38.3 \%$ pass through the slit as show in Fig. 6. Defocusing is only practical in short-foca1length sources where the amount of mass discrimination is sma11. In long-focal-length sources, defocusing for uniform sensitivity causes a severe ion intensity loss and the output is not useful for analytical measurements. 


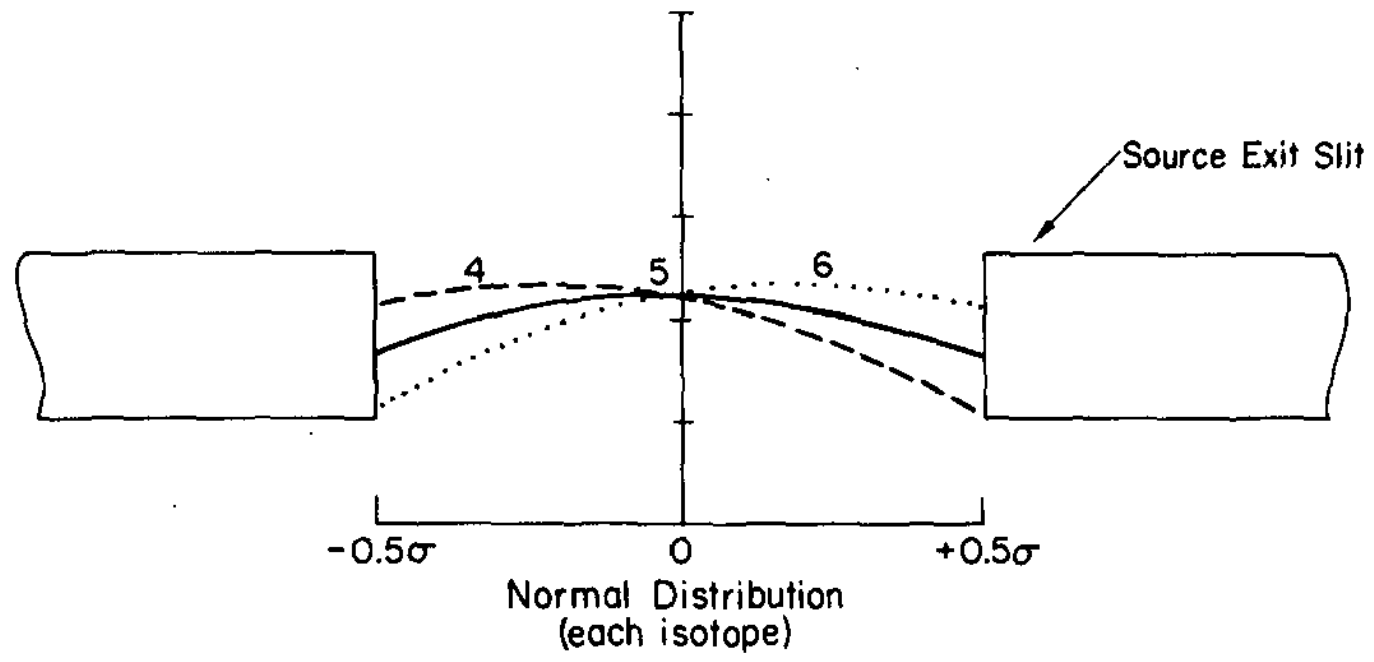

FIGURE 6. Deconvoluted Ion-Beam Profile Showing Effect of Defocusing

\section{Ion Source Design Parameters}

As shown in the analyzer section of this paper, the minimum practical ion beam width of about 50 to $75 \mu \mathrm{m}$ limits the choice of mass analyzers to either large-radius $(\$ 30 \mathrm{~cm})$ single-focusing analyzers or double-focusing analyzers of like performance. Instrument designers have been reluctant to use short-focallength ion sources with large-radius analyzers because these analyzers have smal1 acceptance angles. Long-foca1-1ength sources have been preferred because they have inherently small divergence and are easier to design. However, Loveless and Russe $11^{2}$ have shown that a short-focal-length, strong-focusing ion source with very high transmission can be designed to match the acceptance angle of a large-radius magnetic analyzer. Loveless $^{4}$ and Werner ${ }^{5}$ have both developed computer programs to evaluate ion-source designs. And, Sakudo and-coworkers ${ }^{6}$ have developed a display device that permits ion sources to be studied directly. Werner ${ }^{7}$ found that when the acceleration voltage is changed to scan the mass spectrum, the ion-extraction volume changes. This is the major cause of mass discrimination in sources of very short focal length. This effect is eliminated when the accelerating voltage is held constant and the mass spectrum is scanned magnetically.

To produce ion beams of $1 \times 10^{-9} \mathrm{~A}$, the ion source must use the sample very efficiently. The ionization chamber should be sealed as effectively as possible to raise the chamber pressure and maintain a high sample density in the path of the electron beam. This is done by making the ionization chamber exit-slit and the electron entrance-slit as small as practical, sealing 
the sample inlet line to the chamber with a ball or conical joint, and enclosing the trap within the ionization chamber. The highest practical accelerating voltage, about $10 \mathrm{kV}$, should be used because ion extraction and utilization are more efficient at high voltages and the ion beam is more nearly monochromatic. Z-focusing may be required to optimize the injection of the ion beam into the mass analyzer for efficient transmission to the ion collector.

\section{Trimer Production}

Trimer formation is proportional to the square of the pressure in the ionization chamber. The sample pressure required to obtain an ion bean of $1 \times 10^{-9}$ A may be high enough to cause significant trimer production. However, at a resolution of 2,000, trimer formation would not interfere with the analysis of D-T mixtures because $\mathrm{D}_{3}{ }^{+}$, the only interfering trimer that could be produced in any quantity, is completely resolved from $\mathrm{T}_{2}{ }^{+}$. In most other types of mixtures, the formation of monoisotopic trimers probably would be favored. It is unlikely that significant amounts of the polyisotopic trimers would be produced. Therefore, although low trimer formation is a desirable goal, it is not absolutely essential to the success of a high-resolution mass spectrometer.

\section{Source Construction Materials}

The ion source must be memory-free and must not equilibrate the isotopic species in the sample. Memory is a function of the materials of construction. Satisfactory materials are Nichrome $V^{*}$ for the metal parts and Coors AD94 alumina for the insulators. Nichrome $V$ will take a high surface polish, has a low surface area, and retains these characteristics even at high temperatures. Coors AD94 and alumina is very dense, does not absorb chemicals during cleaning, does not degas when subjected to the filament heat during operation, and maintains usable electrical properties at high temperatures (W. R. Shields, W. R. Shields \& Associates, private comnunication). Stainless steel, copper, aluminum, and glass are less desirable and should be avoided.

Sample equilibration occurs on heated surfaces and on catalytic surfaces. Contact with the heated filament can be avoided by making the electron entrance hole small (already required for high sensitivity). All the metal parts of the ionization chamber can be kept reasonably cool by making them massive enough to conduct away excess heat. Catalytic materials and materials that form catalytic surface films must not be used.

* Trademark of Driver-Harris Company, Harrison, NJ. 
Test of Short-Focal-Length Concept

To confirm the advantages of a short-focal-length ion source, the Savannah River Laboratory (SRL) obtained a 10-mm focal-1ength Nier-type source that is used commercially on large-radius instruments and installed it on a $35-\mathrm{cm}$-radius magnetic analyzer. As reported in Reference 8 , the test was successful. Mass discrimination was small and could be eliminated by defocusing the source slightly. The sensitivity loss on defocusing was only about $10 \%$. Sensitivity was about one-third that of a low-resolution instrument and could have been improved by reducing the size of the ionization chamber exit-slit (which was about $1 \mathrm{~mm}$ wide), enclosing the trap, and reducing the size of the electron entrance hole. Resolution was about 1400 and the abundance sensitivity of the system was about 10,000 for the $D_{2}-H T$ doublet at mass 4 . No trimer formation was observed. A small amount of sample equilibration was observed. It was probably caused by sample contact with the heated filament. A smaller electron entrance hole should eliminate the equilibration problem.

Proposed Virtual-Image Source

R. D. Craig, VG-Micromass Ltd. (Winsford, Cheshire, England), recentiy suggested a more complete solution to the mass discrimination problem. He proposed building a $30-\mathrm{cm}$-radius mass spectrometer with a short-focal-length, virtual-image ion source (R. D. Craig, private communication). This source is a refined version of a virtual-image source currently used on small VG-micromass mass spectrometers (Figure 7).

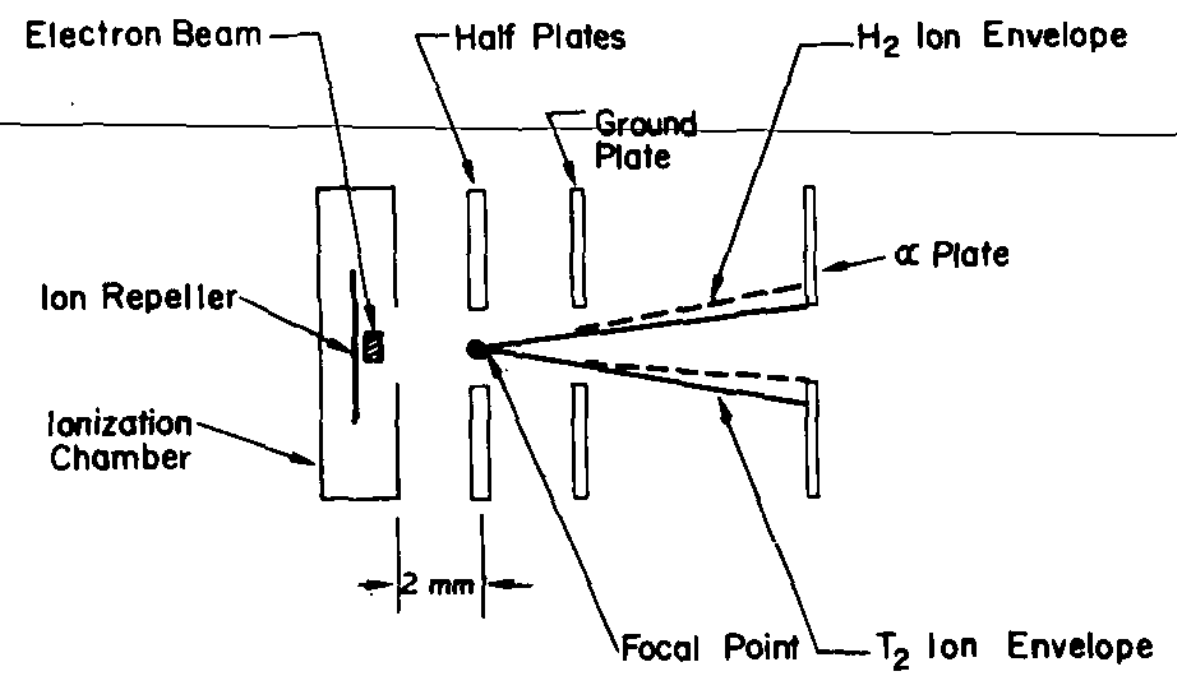

FIGURE 7. Virtua1-Image-Type Ion Source 
In this virtual-image source, a sharply defined image $50 \mu \mathrm{m}$ wide would be formed about $2 \mathrm{~mm}$ from the ionization chamber in the space between two half plates. This image would be the object image of the mass analyzer. A 300-Gauss magnetic field would collimate the electron beam. The mass $2-6$ separation at the focal point would be about $5 \mu \mathrm{m}$ which is only one-eighth of that of the $10-\mathrm{mm}$ focal-length Nier-type ion source that was tested at SRL. The exit slit would be about $250 \mu \mathrm{m}$ wide, in contrast to the 50 $75 \mu \mathrm{m}$ sxit-slit width in a conventional Nier-type source, and would not cause any mass discrimination. An angular acceptancedefining plate (alpha plate) with a $250-\mu m$ wide slit would limit the width of the ion beam entering the mass analyzer. The alpha plate would be far enough from the focal point for the ion beam to be sufficiently defocused to eliminate any measurable mass discrimination. The proposed virtual-image source should be at least 30 times more sensitive than the $10-\mathrm{mm}$ focal-length Nier-type source tested at SRL.

\section{Analyzer}

The mass analyzer should not require any significant development effort because satisfactory mass analyzers exist. The mass analyzer must have high transmission, good abundance sensitivity, a sharp focus, and must be large enough to attain the required resolution with a 50-75 m incident ion beam. A low-impedence, high-current electromagnet should be used to permit rapid magnetic scanning.

The resolving power, $R$, of a single-focusing mass spectrometer is given by the expression

$$
R=\frac{R_{m}}{W_{b}+S_{c}}
$$

where $R$ is the radius of curvature of the analyzer, $w_{b}$ is the width of the ion beam at the collector, and $S$ is the width of the collector slit. ${ }^{9}$ For accurate ion intensisity measurements, the whole ion beam must be measured at one time. The minimum useful width of the collector slit equals the ion bean width, $W_{b}=S_{c}$. Therefore, the minimum radius of a single-focusing magnetic analyzer needed for a resolution of 2,000 with a 75-m ion beam width is $30 \mathrm{~cm}$. Good $30-\mathrm{cm}$-radius analyzers are readily available. 


\section{Abundance Sensitivity}

Ruedenauer showed that gas scattering is a limiting factor in attaining good abundance sensitivity. ${ }^{18}$ The analyzer must be operated at a low enough pressure to minimize scattering. The minimum abundance sensitivity considered acceptable for the $D_{2}-\mathrm{HT}$ doublet is about 10,000 and 20,000 is a desirable goal. An abundance sensitivity of 20,000 can be attained at reasonable analyzer pressures. For example, at an analyzer pressure of $2 \times 10^{-8}$ torr, the gas scattering limit for the $\mathrm{D}_{2}$ - HT doublet is about $50,000-100,000$.

In the test reported in Reference 8 , an abundance sensitivity of 10,000 was attained for the $\mathrm{D}_{2}-\mathrm{HT}$ doublet position at mass 4 with a 35-cm-radius single-focusing mass analyzer operating at a pressure of about $2 \times 10^{-8}$ torr.

Low pressure is not enough to assure good abundance sensitivity. Good magnetic analyzer design is also required. Sectortype analyzers have been shown to have good abundance sensitivity, but no spectra have been reported that show good abundance sensitivity in other types of mass analyzers. One Dempster-type instrument was reported to have poor abundance sensitivity, regardless of pressure (L.A. Dietz, Knolls Atomic Power Laboratory, private communication). Appropriate large sector-type analyzers are readily available and there is no need to develop another analyzer type.

\section{Analyzer Types}

Either single-focusing or double-focusing analyzers can be used. The former has the advantage of simplicity while the latter provides better resolution and probably better abundance sensitivity. Judging from our recent work ${ }^{8}$, and previous work with the same instrument in other mass ranges, an abundance sensitivity of about 20,000 for the $D_{2}-H T$ doublet (the design goal of this work) is attainable with a large-radius single-focusing mass analyzer.

A double-focusing mass analyzer focuses ions according to energy as well as mass and both resolution and abundance sensitivity are enhanced. In the conventional double-focusing arrangement [electrostatic analyzer (ESA) before the magnetic analyzer], the ESA acts as an extension of the ion source and introduces mass discrimination if any stray magnetic fields are present. Even the earth's magnetic field is strong enough in many locations to partially separate the hydrogen isotopes as they traverse a large-radius ESA. For this reason, the ESA must be very carefully shielded. Because stray magnetic fields are also a problem in ultra high-resolution instruments, the manufacturers usually shield them carefully. For example, 
the ESA of the MS50 built by AEI Scientific Equipment Company of Manchester, England is shielded with soft iron, so the residual magnetic field is less than 100 milligauss (S. Evans, AEI

Scientific Company, private communication).

\section{Electrostatic Filters and Analyzers}

In the conventional arrangment, the ESA eliminates energy differences that occur in the ion source. It does not eliminate ions that lose energy or are scattered by collisions in the magnetic analyzer and the best abundance sensitivity is not attained. Reversed double-focusing geometry is preferred because energy focusing occurs after mass separation and immediately before the ion collector. Mass discrimination is no longer a problem, and the defining slit that is usually put between the magnetic and electrostatic analyzers eliminates scattered ions. The abundance sensitivity attained should be close to the gas scattering limit of about 50,000 .

As an alternative to the complex double-focusing geometry, an energy filter or a cylindrical ESA can be used with a singlefocusing magnetic mass analyzer to improve abundance sensitivity.

Electrostatic filters located after the exit slit of a singlefocusing mass analyzer are also used to improve abundance sensitivity. Axial energy filters ${ }^{1,12}$ improve abundance sensitivity substantially by eliminating ions of below-average energy. The filters do not eliminate ions that have higher-than-average energy. These filters also act as lenses and can improve the resolution of the instrument. Unfortunately, unless they are well designed, they can also alter the peak shape and interfere with quantitative measurements.

A large-radius, single-focusing mass spectrometer equipped with an axial energy filter should have an abundance sensitivity for the $\mathrm{D}_{2}-\mathrm{HT}$ doublet between 20,000 (probably attainable without a filter) and 50,000 (the estimated gas scattering limit).

Symmetrical, 90-degree, cylindrical ESA's effectively control the energy spread of the ion beam in high abundance-sensitivity tandem mass spectrometers. ${ }^{13}, 14$ The design of these analyzers is highly refined. Each end of the analyzer is terminated by an apertured Herzog plate to define the electrostatic field boundary, tapered rose shims are used to improve field uniformity, ${ }^{15}$ and the upper and lower supporting plates can be operated at different potentials to focus the analyzer. ${ }^{16}$ These radial analyzers reject all ions with energies higher or lower than a certain pass band, and focus those that are within the band. The abundance sensitivity of a large-radius single-focusing instrument with a 
cylindrical ESA of this design would be expected to approach the estimated gas-scattering limit of about 50,000 for the D-HT doublet. The instrument should be almost as simple to operate as a single-focusing instrument.

Col 1ector

The ion collector must collect all of the incident ions without 1oss. Secondary ions and electrons (resulting from incident primary ion impact on the flight tube, exit slit, or collector) must be shielded out or suppressed if accurate ion measurements are to be made. A deep-bucket Faraday cage similar to that used in the National Bureau of Standards (NBS) ion collector ${ }^{17,18}$ is required to hold all the incident ions without backscatter. The interior of the cage must be clean and free of insulating deposits that would become charged under ion bombardment and cause inaccurate measurements.

Secondary electron production at the collector is especially severe with the light elements and the NBS cubic suppressor system does not work with 8-10 kV incident hydrogen ions. In the work reported in Reference 8, a "brute-force" system consisting of a combination of electrostatic and magnetic fields proved to be effective.

Electron multipliers and Daly-type scintillation ion detectors are limited to ion currents less than $1 \times 10^{-12} \mathrm{~A}$, and they exhibit mass discrimination. They are not useful for making accurate measurements of intense ion beams $\left(>1 \times 10^{-11} \mathrm{~A}\right)$.

\section{Electronics}

The electronic power supplies, control circuits, and measuring devices-should-be-of-all solid state_construction for high reliability. The circuits must be very stable if the desired precision and accuracy are to be attained. Adequate electronic units exist for all parts of the mass spectrometer and no development effort is required.

A magnetic field controller with a Hall-effect-type probe must be used to control the magnetic analyzer. The field should be stabilized within 1 part in 50,000 and must be resettable within that error limit. The ion-accelerating voltage should also be stable within 1 part in 50,000. Magnetic peak switching and scanning are both necessary because peak switching between the $D_{2}$ and HT mass positions does not appear to be within the current state-of-the-art. Operation of the instrument would probably require switching from unit mass to unit mass and scanning the peaks at each mass region. Voltage scanning introduces mass discrimination and is not useful. 
Fast solid-state electrometer amplifiers that are precise and accurate within $0.1 \%$ and are sensitive to signals less than $1 \times 10^{-14} \mathrm{~A}$ are available. The random noise of a typical satisfactory amplifier is shown in Figure 8. Vibrating reed electrometer amplifiers have lower noise and could be used; however, they are undesirable because they have longer time constants and would make it difficult to attain the required analysis rates. The added cost is not justified for this application.

The maximum value of the input resistor to the amplifier should be limited to $1 \times 10^{11}$ ohms because the linearity and stability of higher-valued resistors exceeds $0.1 \%$, and detector time constants become too long for the desired scanning speeds.

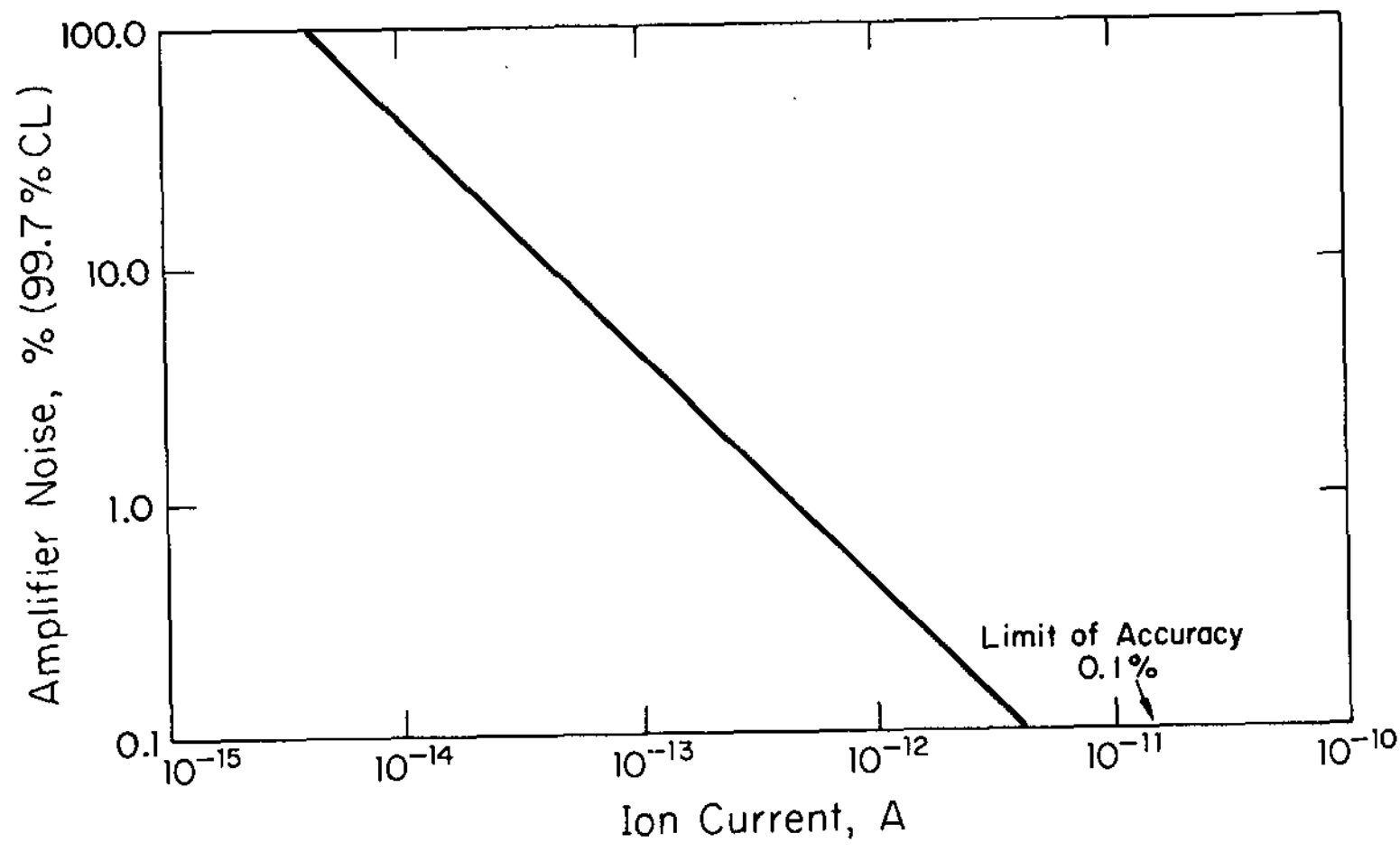

- FIGURE 8. Amplifier Performance

Vacuum System

The vacuum envelope should be made entirely of Type 304 stainless steel. It should be stress-relieved and annealed at intervals during its fabrication. All welds, especially in the fabrication of the flight tube, should be properly quenched to prevent the formation of magnetic areas (W. R. Shields, W. R. Shields $\&$ Associates, private communication). Stainless steel is preferred 
because its outgassing rate is less than that of any other commonly used material. ${ }^{9}$ It is not subject to corrosion, is bakeable to at least $400^{\circ} \mathrm{C}$, and is dimensionally stable.

Diffusion pumps with appropriate traps must be used to evacuate the ion source and the sample inlet system. Ion pumps may be used to evacuate the flight tube and collector where the gas load is comparatively low. Ion pumps may also be used to maintain source and inlet pressure during periods of standby operation. Ion pumps tend to regurgitate the hydrogen isotopes and are unsatisfactory for the high hydrogen gas loads encountered in the routine operation of the ion source and the sample system. Turbomolecular pumps are attractive because they are inherently clean. Unfortunately, their hydrogen compression ratio is very low and they would have to be backed by diffusion pumps for use in this application.

The mechanical pumps used for rough-pumping the vacuum system and backing the diffusion pumps must be isolated from the system by either optically dense or molecular sieve traps to prevent the migration of mechanical pump oil into the vacuun system.

\section{ANALYTICAL ACCURACY}

A mass spectrometer that meets the above design criteria should be able to analyze directly mixtures of the hydrogen and helium isotopes, regardless of composition or equilibrium state. Analyses should be accurate within \pm 0.5 relative $(99.7 \% \mathrm{CL}$ ) with pure-gas standardization and within $\pm 0.3 \%$ relative $(99.7 \% \mathrm{CL})$ when standardized with mixed standards that are accurate within $\pm 0.15 \%$ relative $(99.7 \% \mathrm{CL})$. Minor components $(<0.1 \%)$ can be measured accurately within $2-3 \%$. 


\section{REFERENCES}

1. W. M. Jones. "Thermodynamic Functions for Tritium Deuteride. The dissociation of Tritium Particles. Equilibrium among the Isotopic Hydrogen Molecules." J. Chem. Phys. 17, 1062 (1949).

2. R. Schott and G. Beau. "Quantitative Analysis of Hydrogen Isotopes by Mass Spectrometry." Method. Phys. Anal. 7, 165 (1971).

3. A. J. Loveless and R. D. Russe11. "A Strong-Focusing Lens for Mass Spectrometer Ion Sources." Int. J. Mass Spectrom. Ion Phys. 3, 257 (1969).

4. A. J. Loveless. Ion Trajectories in Mass Spectrometer Ion Sources. "M.Sc. Thesis, University of Toronto, Toronto, Canada, 1967.

5. H. W. Werner. "A Study on a Mass Spectrometer for Source by Means of Computer-Calculated Ion Trajectories." J. Phys. E. 7, 115 (1974).

6. N. Sakudo, I. Kanomata, H. Tsuyama and E. Mitani. "Display Device for Ion Beam Profile." Rev. Sci. Instrum., 47, 1288 (1976).

7. H. W. Werner. "A Study on Mass Discrimination in a Magnetic Sector Mass Spectrometer." J. Mass Spectrom. Ion Phys. 14, 189 (1974).

8. R. B, Ferguson and P. Chastagnex. "High Resolution Mass Spectrometer for Accurate Analysis of Mixture of Hydrogen and Helium Isotopes." Int. J. Mass Spectrom. Ion Phys. 24, 403 (1977).

9. J. Roboz. Introduction to Mass Spectrometry. p. 56, John Wiley and Sons, New York, 1968.

10. F. G. Ruedenauer. "Gas Scattering as a Limit to Trace Sensitivity in Analytical Mass Spectrometers." Rev. Sci. Instrm. 41, 1487 (1970).

11. N. J. Freeman, N. R. Daly, and R. E. Powe11. "Retardation Lens Used to Improve the Abundance Sensitivity of a Mass Spectrometer." Rev. Sci. Instrum. 38, 945 (1967). 
12. K. A. Kaisex and C. M. Stevens, Ion-Retarding Lens to Improve the Abundance Sensitivity of Tandem Mass Spectrometers. USAEC Report ANL-7393, Argonne National Laboratory, Argonne, I11. (1967).

13. F. A. White, F. M. Rourke, and J. C. Sheffield. "A Three Stage Mass Spectrometer." AppZ. Spectry. 12, 46 (1958).

14. C. R. Lagergren and J. J. Stoffels. "A Computer-Controlled, Three Stage Mass Spectrometer." Ion Phys. 3, 429 (1970).

15. L. A. Dietz. "Electrostatic Field Correction. Using Tapered Rose Shims." Rev. Sci. Instrum. 32, 859 (1961).

16. H. Matsuda. "Electrostatic Analyzer with Variable Focal Length." Rev. Sci., Instrum., 32, 850 (1961).

17. W. R. Shields, Ed., NBS Technical Note 277, U. S. Government Printing Office, Washington, DC, 1966.

18. W. R. Shields, Ed., NBS Technical Note 426, U. S. Government Printing Office, Washington, DC, 1967.

19. L. Holland, W. Steckelmacher, and J. Yarwood, Eds., p 4, 5 in Vacuum Manual. E \& F.N. Spon. London, 1974. 